\title{
The Optimal Depth of Central Venous Catheter by Using Transesophageal Echocardiography for Pediatric Patients
}

\author{
Department of Anesthesiology, Seoul National University College of Medicine, Seoul, Korea \\ Teo Jeon Shin, M.D., Seung Joo Yoon, M.D., Chongdoo Park, M.D., \\ Chong Sung Kim, M.D., and Seong Deok Kim, M.D.
}

\begin{abstract}
Background: Incorrect positioning of central venous catheter leads to serious complications. To prevent these complications, catheter tip should be at the superior vena cava and right atrial junction.

Methods: We studied 60 right internal jugular catheterizations in infants and children undergoing surgery for congenital heart disease. To confirm the optimal depth of central venous catheter, we measured the distance from the skin puncture site to subclavian vein-right atrial junction using transesophageal echocardiography.

Results: The measured distance highly correlated with the patient height. Based on these data, following guideline could avoid intra-atrial placement in $94 \%$ of the time: optimal depth of insertion $(\mathrm{cm})=2.5+(0.06 \times$ height $)$.

Conclusions: We postulate that initial using a simple practical guideline could prevent malposition of central venous catheter. (Korean J Anesthesiol 2005; 48: S 11 4)
\end{abstract}

Key Words: central venous catheterization, internal jugular vein, transesophageal echocardiography.

\section{INTRODUCTION}

Percutaneous central venous catheter (CVC) insertion in pediatric patients for congenital heart disease is mandatory for hemodynamic monitoring and drug delivery to central circulation. $^{1,2)}$ However, this procedure carries the risk of complications such as incorrect positioning of the catheter, hemothorax, pneumothorax, and perforation of mediastinal vessels. ${ }^{3,4)}$ To decrease the incidence of this complication, accurate positioning of catheter tip near to superior vena cava and right atrium (SVC-RA) junction has been proposed.

In pediatric patients, there have not been adequate guidelines available for the optimal depth of CVC. There have been several studies with regard to the optimal depth of CVC. ${ }^{5,6}$ However, chest radiograph was used to determine the CVC position in these results. The assessment of catheter tip position using chest radiograph is inaccurate. ${ }^{7)}$ In contrast, transeso-

Received : March 23, 2005

Corresponding to : Chongdoo Park, Department of Anesthesiology and Pain Medicine, Seoul National University Hospital, 28 Yeongeondong, Jongno-gu, Seoul 110-744, Korea. Tel: 82-2-2072-2065, Fax: 82- 2-747-5639, E-mail: cdpark@snu.ac.kr

This is a thesis for degree of Doctor of Master in Medicine. phageal echocardiography (TEE) has been shown to the placement of catheter tip at SVC-RA junction. ${ }^{8)}$ Thus, we measured the distance from the skin puncture site to SVC-RA junction using the TEE to suggest ideal catheter insertion depth through right IJV in pediatric patients.

\section{MATERIALS AND METHODS}

Institutional Ethics Committee approval and informed parental consent were obtained. Pediatric patients scheduled for congenital heart surgery were enrolled. Patients with any extracardiac vascular abnormality or rotational abnormality were excluded. $60 \mathrm{CVC}$ cannulations were studied. The demographic data of the patients are listed in Table 1 .

After the introduction of general anesthesia, the patients were placed in the Trendelenburg position, with the head rotated $45^{\circ}$ to the left. A high approach to IJV as described by Andropoulos et $\mathrm{al}^{5)}$ was performed on the right IJV. If the CVC could not be advanced to the SVC-RA junction, subclavian vein (SVC) catheterization was attempted, and the patient was excluded from the study. After performing CVC cannulation, the same anesthesiology staff operated the TEE probe to obtain a longitudinal view of the atria and the SVC, and 
observe SVC-RA junction. The tip was identified by directly visualizing many hyperechogenic microbubbles quickly flowing out of the distal catheter after a rapid flush of saline. Using the TEE, the initial position of the catheter tip was defined as the SVC-RA junction if the tip was located at the superior border of the crista terminalis. After locating the tip of CVC was located at SVC-RA junction, a nurse blinded to the experiment measured the length of the CVC beneath the skin using the depth indicator on the CVC.

All data including patient height, age, and weight were collected. Plots of distance from skin to the SVC-RA junction and age or height or weight were made. Simple linear regression analysis was performed by the least square method. Multiple linear regression analysis was performed to identify independent factors affecting the distance from the skin to the SVC-RA junction. Statistical significance was accepted at $\mathrm{P}<0.05$.

\section{RESULTS}

The TEE confirmed that all catheter tips were located at the SVC-RA junction. None of the catheter tips were located

Table 1. Demographic data

\begin{tabular}{lc}
\hline Age $($ month $)$ & $35.0 \pm 43.4$ \\
Sex $(\mathrm{M} / \mathrm{F})$ & $39 / 24$ \\
Height $(\mathrm{cm})$ & $87.7 \pm 24.6$ \\
Weight $(\mathrm{kg})$ & $13.1 \pm 8.0$
\end{tabular}

The values are expressed as mean \pm SD except sex.

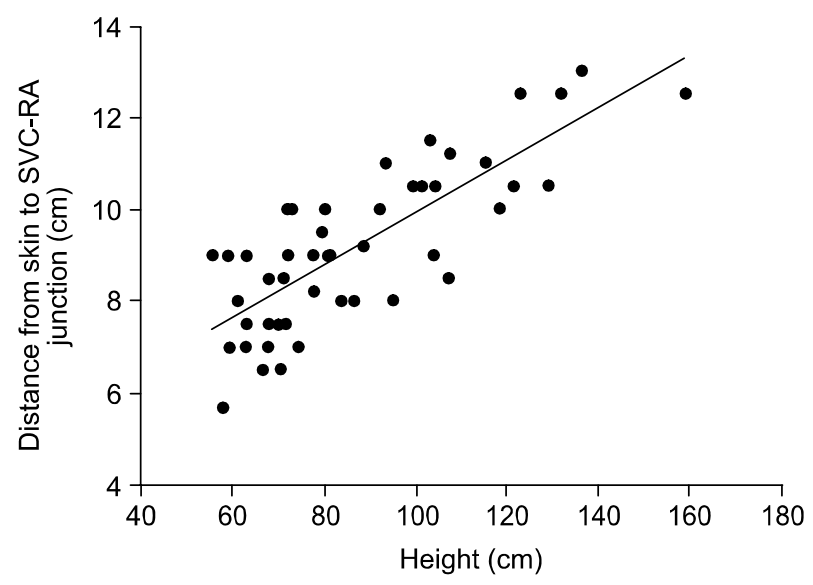

Fig. 1. The plot between distance from skin to subclavian vein-right atrial (SVC-RA) junction and height $\left(\mathrm{r}^{2}=0.67, \mathrm{P}<0.001\right)$. The distance from skin to SVC-RA junction $(\mathrm{cm})=4.19+0.056 \times$ height $(\mathrm{cm})$. below SVC-RA junction or within RA.

With simple linear regression analysis, the distance from the skin puncture site to the SVC-RA junction showed a higher correlationship with the patient height $(\mathrm{r}=0.81)$ rather than weight $(r=0.76)$ or age $(r=0.60)$ (Fig. 1-3). In multiple regression analysis, when the other two variables were compensated, patient's height only showed significant correlationship with the distance from the skin puncture site to the SVC-RA junction. The distance from the skin puncture site to the SVC-RA junction could be predicted from height using the following.

Distance to SVC-RA junction $(\mathrm{cm})=3.86+(0.06 \times$ height $)$ :

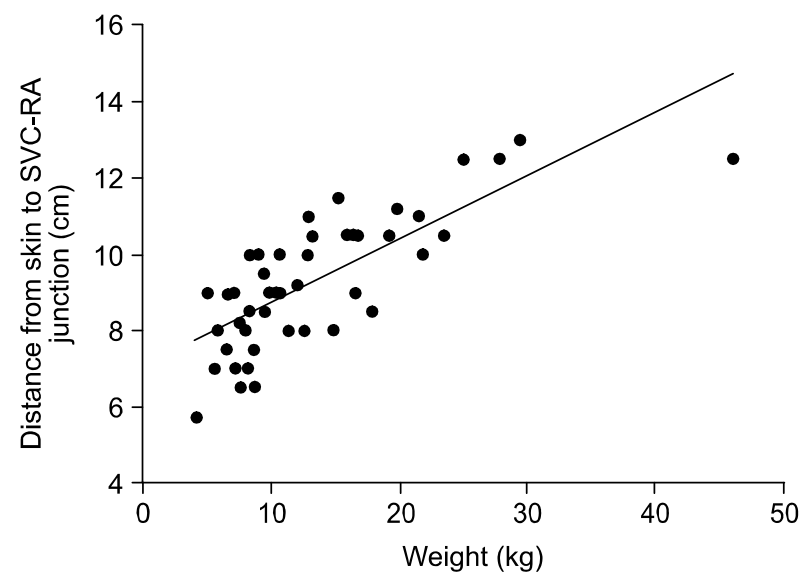

Fig. 2. The plot between distance from skin to subclavian vein-right atrial (SVC-RA) junction and weight $\left(\mathrm{r}^{2}=0.59, \mathrm{P}<0.001\right)$. The distance from skin to SVC-RA junction $(\mathrm{cm})=7.01+0.16 \times$ weight $(\mathrm{kg})$.

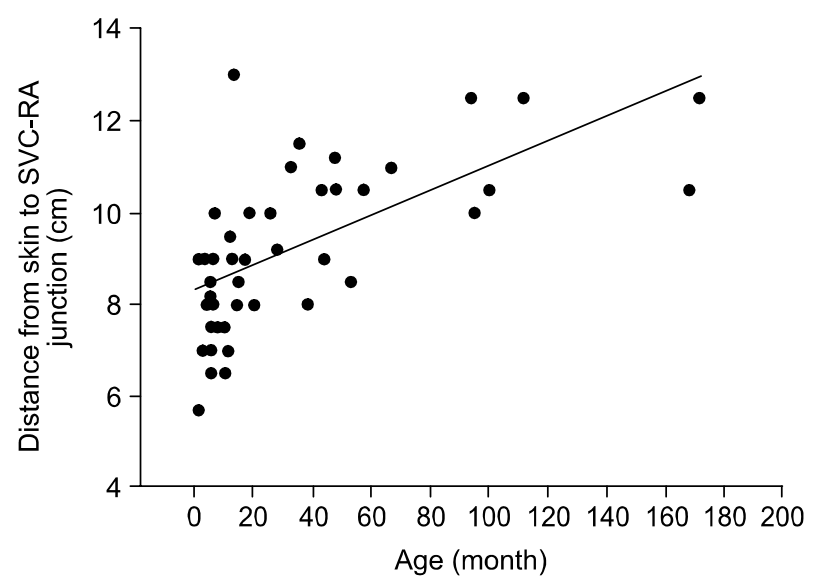

Fig. 3. The plot between distance from skin to subclavian vein-right atrial (SVC-RA) junction and age $\left(\mathrm{r}^{2}=0.37, \mathrm{P}<0.01\right)$. The distance from skin to SVC-RA junction $(\mathrm{cm})=8.30+0.03 \times$ age $($ month $)$. 


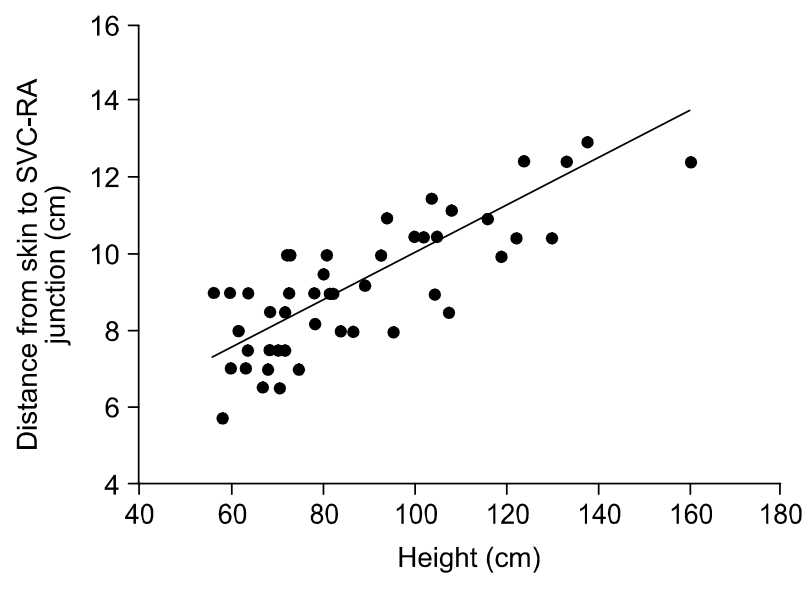

Fig. 4. The distance from skin to subclavian vein-right atrial (SVC-RA) junction was derived from the result of multiple regression analysis $\left(\mathrm{r}^{2}\right.$ $=0.63, \mathrm{P}<0.001)$. The distance from skin to SVC-RA junction $(\mathrm{cm})$ $=3.67+0.06 \times$ height $(\mathrm{cm})$.

$$
\left(\mathrm{r}^{2}=0.63, \mathrm{P}<0.001\right) \text { (Fig. 4) }
$$

Based on the data obtained, we made a formula that predicted that a CVC would be positioned above the RA in $94 \%$ (95\% confidence interval: $90.0-96.7 \%)$ of the patients.

Optimal depth of insertion $(\mathrm{cm})=2.5+(0.06 \times$ height $)$

Lines representing these recommended insertions are included in Fig. 5.

When our guidelines were applied to present data in 60 patients, $56 \mathrm{CVC}$ were found to be above SVC-RA junction. All of 4 CVC found to be in RA were within $1 \mathrm{~cm}$ of the SVC-RA junction.

\section{DISCUSSION}

Optimal positioning of the catheter tip of CVC is a complex and controversial subject. The ideal catheter tip placement in the venous system is mainly related to the catheter function for its intended use. Placing catheter tips into the right atrium provide optimal performance and superior functional ability compared to catheter tip above the SVC. ${ }^{7)}$ However, there are several reports describing complications attributed to central venous catheter tips positioned within the right atrium. These complications include cardiac perforation, tamponade and cardiac arrhythmia. $^{2-4)}$ With regard to the prevention of fatal complications, the catheter tip should be positioned at the SVC-RA junction or in the SVC.

Percutaneous puncture of right IJV has been the preferred method. IJV cannulation provides intraoperative accessibility to

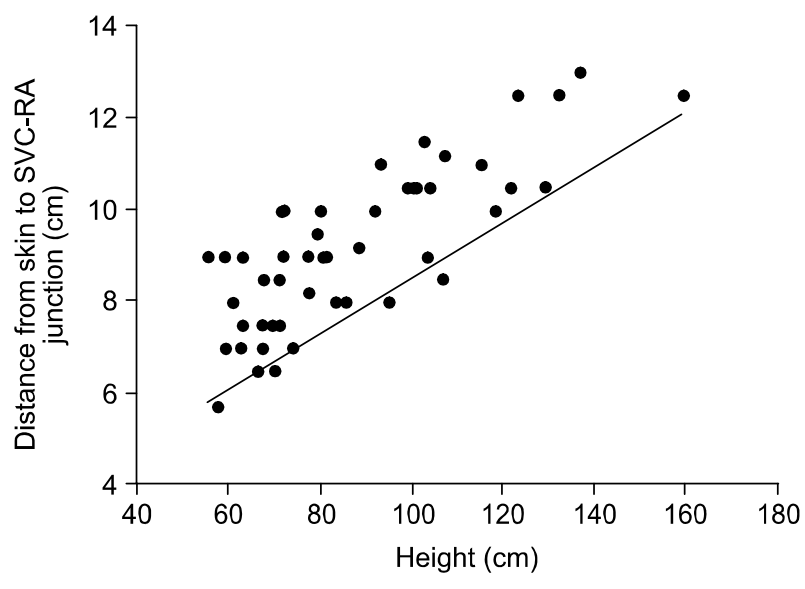

Fig. 5. The plot of patient height versus distance from catheter insertion site above the junction of subclavian vein-right atrium (SVC-RA) and RA for right internal jugular catheter. The solid line represents our recommendation for initial length of the catheter insertion. Optimal depth of insertion $(\mathrm{cm})=2.5+0.06 \times$ height $(\mathrm{cm})$.

the anesthesiologist and high success rate. The right IJV is preferred because the risk of pneumothorax or chylothorax is lower than right SCV. ${ }^{9)}$ However, there have been a few studies that tried to determine the optimal depth in IJV in pediatric patients. For these reasons, we choose the main cannulation site as right IJV in our study.

In several studies, postoperative chest $\mathrm{X}$-ray was used for the assessment of the ideal location of catheter tip. ${ }^{5,6)}$ However, radiographic borders of SVC and RA border has not been well defined and the distance from the catheter tip to SVC-RA junction is magnified in the chest radiograph. This magnification of image must be taken into consideration when measuring the distance accurately in the chest radiograph. The determination of the catheter tip position with the chest radiograph is often imprecise and depends on interobserver variability. ${ }^{10)}$ Meanwhile, the TEE technique identifies catheter tip location in the SVC by direct two-dimensional visualization of the vessel. And an adjustment to catheter position can be made at the time of replacement. It is reported that the TEE improve success rate of proper CVC placement. ${ }^{11)}$ Taking these findings into consideration, the TEE is more advantageous to confirm the catheter's location compared with the chest radiograph.

Andropoulos et $\mathrm{al}^{5)}$ suggested a guideline for proper depth of $\mathrm{CVC}$ in pediatric patients. Their result is slightly differently from our results. The reasons for this discrepancy may be as follows. (1) Andropoulos et al combined the results of the IJV 
and SCV. However, the distance from the skin to SVC-RA junction is shorter for IJV compared with SCV. ${ }^{12)}$ Since we only derived the equation from the result of IJV, our formula is shorter than that of Andropoulos result. And this is compatible with our result that is shorter than his result. (2) In our study, we use TEE, and not the chest radiograph for the determination of the distance from the skin to SVC-RA junction. As previously mentioned, it is known that the TEE provides a more sensitive assessment of catheter tip than the chest radiograph. ${ }^{11)}$ The possibility of the distance to SVC-RA junction being measured inaccurately could not be excluded in his study.

However, there are some limitations to our study. Our formula for CVC insertion is only applied to a cephalad approach to IJV. If other methods such as a lower approach to IJV or SCV cannulation are used, our formula cannot be used. Second, our formula, which is mainly based on the patient height, cannot be applied to children whose height is extremely below or above average. However, there were no patients extremely below or above average height in our study. When our formula is used in clinical practice, it must be taken into consideration.

In conclusion, we postulate that initial using a simple practical guideline could prevent malposition of CVC.

\section{REFERENCES}

1. Hayashi Y, Maruyama K, Takaki O, Yamauchi J, Ohnishi Y, Kuro M: Optimal placement of the CVC catheter in pediatric cardiac patients. Can J Anaesth 1995; 42: 479-82.
2. Fischer GW, Scherz RG: Neck vein catheters and pericardial tamponade. Pediatrics 1973; 52: 868-71.

3. Dane TE, King EG: Fatal cardiac tamponade and other mechanical complications of central venous catheters. Br J Surg 1975; 62:6-10.

4. Defalque RJ, Campbell C: Cardiac tamponade from central venous catheters. Anesthesiology 1979; 50: 249-52.

5. Andropoulos DB, Bent ST, Skjonsby B, Stayer SA: The optimal length of insertion of central venous catheters for pediatric patients. Anesth Analg 2001; 93: 883-6.

6. Kim KO, Jo JO, Kim HS, Kim CS: Positioning internal jugular venous catheters using the right third intercostal space in children. Acta Anaesthesiol Scand 2003; 47: 1284-6.

7. Vesely TM: Central venous catheter tip position: a continuing controversy. J Vasc Interv Radiol 2003; 14: 527-34.

8. Chu KS, Hsu JH, Wang SS, Tang CS, Cheng KI, Wang CK, et al: Accurate central venous port-A catheter placement: intravenous electrocardiography and surface landmark techniques compared by using transesophageal echocardiography. Anesth Analg 2004; 98 : 910-4.

9. Miller R: Anesthesia. 5th ed. Philadelphia, Churchill Livingstone. 2000, pp 1144-5.

10. Aslamy Z, Dewald CL, Heffner JE: MRI of central venous anatomy: implications for central venous catheter insertion. Chest 1998; 114: 820-6.

11. Andropoulos DB, Stayer SA, Bent ST, Campos CJ, Bezold LI, Alvarez M, et al: A controlled study of transesophageal echocardiography to guide central venous catheter placement in congenital heart surgery patients. Anesth Analg 1999; 89: 65-70.

12. Andrews RT, Bova DA, Venbrux AC: How much guidewire is too much? Direct measurement of the distance from subclavian and internal jugular vein access sites to the superior vena cava-atrial junction during central venous catheter placement. Crit Care Med 2000; 28: 138-42. 